\title{
ESTUDIO MORFOLÓGICO Y MORFOMÉTRICO DEL CEREBRO DEL BAGRE TIGRITO Pimelodus pictus (Pimelodidae) Steindachner (1876)
}

Fecha de recepción: 19 de enero de 2013 • Fecha de aceptación: 25 de marzo de 2013

\section{MORPHOLOGICAL AND MORPHOMETRICAL STUDY OF BAGRE \\ TIGRITO Pimelodus pictus (Pimelodidae) Steindachner (1876) BRAIN.}

\author{
Laura Sofía Valderrama Vizcaino ${ }^{1,2} \bullet$ Laura Rincón ${ }^{1,2} \bullet$ Martha Janneth Obando Bulla ${ }^{1,2,5} \bullet$ Edwin Gómez-Ramírez \\ - Hernán Hurtado Giraldo ${ }^{3,4}$
}

\section{RESUMEN}

En el presente trabajo se realizó una descripción de la anatomía externa del cerebro de Pimelodus pictus, identificando las principales estructuras que lo conforman. Posteriormente, se realizó un análisis morfométrico de algunas regiones del cerebro (bulbos olfativos, hemisferios telencefálicos, lóbulos ópticos, corpus cerebelar, válvula cerebelar, lóbulos vagales, lóbulos faciales y lóbulos inferiores) para determinar diferencias en tamaño, realizando mediciones de área y longitud. De acuerdo a los resultados obtenidos, el cerebro de $P$. pictus presenta una morfología acorde con el patrón de organización general descrito para teleósteos pertenecientes al orden Siluriformes, siendo la válvula cerebelar la estructura más grande del cerebro. De acuerdo a los resultados morfométricos obtenidos esta especie podría clasificarse como generalista, debido a que parece utilizar primordialmente tres sistemas sensoriales para explorar su hábitat: el tacto, la vista y el gusto. El tacto, debido a la válvula cereberal de gran tamaño, la cual está relacionada directamente con la presencia de la línea lateral, que es un órgano sensorial cutáneo. La visión, teniendo en cuenta que los lóbulos ópticos de $P$. pictus presentaron un gran tamaño lo que puede deberse a una especialización visual de esta especie de acuerdo con el ambiente en el que se desenvuelve. Y por ultimo, el gusto, porque los lóbulos vagales y faciales de P. pictus en comparación con otras especies de peces son grandes, esto por la presencia de barbillones, que son estructuras especializadas en integrar señales cutáneas y gustativas.

Palabras clave: teleósteos, sistema nervioso central, neuroecología, Pimelodus pictus.

\footnotetext{
1 Laboratorio de Embriología, Grupo de Ictiología, Facultad de Ciencias Básicas y Aplicadas, Universidad Militar Nueva Granada.

2 Biologo, Egresado del Programa de Biología Aplicada, Facultad de Ciencias Básicas, Universidad Militar Nueva Granada.

3 Biólogo, Especialista en Acuicultura Continental, Magister en Acuicultura, Asistente de investigación y Docente de Catedra, Programa de Biología Aplicada, Facultad de Ciencias Básicas, Universidad Militar Nueva Granada.

4 Docente, Programa de Biología Aplicada, Facultad de Ciencias Básicas, Universidad Militar Nueva Granada.

5 Autor para correspondencia: martha.obando@unimilitar.edu.co
} 


\begin{abstract}
A description of the Pimelodus pictus external brain anatomy was carried out, identifying its main structures. Then a morphometrical analysis of some brain regions were made (olfactory bulbs, telencephalic hemispheres, optic lobes, cerebelar corpus, cerebelar valve, vagal lobes, facial lobes and inferior lobes), in order to find size difference in area and length of these regions. According to our results, P. pictus brain does exhibit the general brain organization pattern described for Siluriform teleosts, with the cerebelar valve as the largest structure. From morphometrical results, this species can be classified as generalist, due to the use of mainly three sensory systems for interaction with it environment: touch, sight and taste. Touch for the large cerebelar valve, directly related with lateral line presence, which is cutaneus sensory organ. Sight, as P. pictus optic lobes have a large size, probably reflecting a visual specialization for its environment. An finally taste, as $P$. pictus vagal and facial lobes are large in comparison with other fish species, due to the preence of barbels wich are cutaneous and taste integration specialized structures.
\end{abstract}

Key words: teleosts, central nervous system, neuroecology, Pimelodus pictus.

\title{
INTRODUCCIÓN
}

Los teleósteos representan el grupo de mayor radiación de los vertebrados, comprendiendo alrededor de 25.000 especies, que presentan gran variación en la complejidad conductual y cerebral, encontrándose diversas adaptaciones tanto interespecífica como intraespecíficamente (Kotrschal et al., 1998; Ishikawa et al., 1999; Wagner, 2001a, b). Por ello, estudiar el cerebro de los teleósteos ha sido de gran interés dado la variación en morfología y tamaño entre estructuras del cerebro, de acuerdo al grado de diversidad filogenética y adaptabilidad funcional de las diferentes especies (Kotrschal et al., 1998; Iwaniuk, 2010).

Además, estos estudios ofrecen una herramienta para relacionar las características del ambiente en el cual vive cada organismo, con el cerebro y los sistemas sensoriales. Esto con el fin de establecer las posibles relaciones entre tipos de hábitats y cómo éstos pueden generar presión selectiva sobre las diferentes facultades sensoriales y otras funciones cerebrales (Kotrschal et al., 1998; Iwaniuk, 2010). Teniendo en cuenta lo anterior, los teleósteos son un buen modelo para realizar investigaciones neurobiológicas, que permitan ampliar el conocimiento sobre las bases neurales que involucran los mecanismos de percepción y acción en su ambiente, y así comprender la historia evolutiva del cerebro y el comportamiento de los vertebrados (Kotrschal et al., 1998; Ishikawa et al., 1999; Wagner, 2001a, b).

El bagre tigrito (Pimelodus pictus) tiene una amplia distribución encontrándose en diversos ambientes. Para Colombia, se localiza en los ríos Meta y Guaviare de la cuenca del río Orinoco, y en el río Caquetá de la cuenca del río Amazonas (Junca et al., 2002; Rodríguez y Mojica, 2005; Moreno et al., 2008; Salas et al., 2006; Aya et al., 2011). En general, habita en aguas cálidas, con temperaturas entre los $22^{\circ}$ a $27^{\circ} \mathrm{C}$. En cuanto a su morfología general, tiene un cuerpo fusiforme con disposición ventral; 
boca en posición ventral con dientes viliformes, una espina en la aleta dorsal, tres pares de barbillones en la boca (mentonianos y maxilares) que se prolongan más allá de la aleta caudal. También cuenta con ojos de gran tamaño que observados dorsalmente interrumpen el perfil lateral del individuo (Dahl, 1971; Galvis et al., 1997; Salas et al., 2006).

Ésta especie se caracteriza por ser de hábitos omnívoros con preferencia sobre restos de peces y material orgánico en descomposición de origen animal (Pérez-Chaparro et al., 2001; Junca et al., 2002; Rodríguez y Mojica, 2005; Olaya et al., 2007). Tiene hábitos crepusculares y utiliza sus barbillones para encontrar sus presas. Presentan un comportamiento territorial con carácter agresivo (Rodríguez y Mojica, 2005; Salas et al., 2006; Moreno et al., 2008). Es un pez reofílico que se desarrolla en ambientes acuáticos laterales donde alcanzan su estado adulto y madurez sexual, para luego migrar por decenas de kilómetros, formar cardúmenes y desovar. Su reproducción se produce cuando las aguas comienzan a subir, entre abril y mayo, presentando desoves asincrónicos y fecundidad total con un promedio de $1.494 \pm 627$ huevos (Pérez-Chaparro et al., 2001 y Junca et al., 2002; Rodríguez y Mojica, 2005). En el trabajo reportado por Mondragón et al., en prensa, se definió la cinética de crecimiento, la supervivencia, algunos parámetros productivos, además de la caracterización de algunas variables fisicoquímicas en sistemas cerrados de recirculación (SCR) de esta especie, con la finalidad de aportar elementos al conocimiento del cultivo.

En cuanto al comercio, P. pictus fue incluida en 1988 en el Programa Nacional para el Desarrollo de la Acuicultura y su subprograma para el cultivo de peces ornamentales, los cuales se desarrollaron con el fin de disminuir la presión pesquera, buscando la creación de paquetes tecnológicos para la producción y el cultivo, debido a su alto valor comercial y gran aceptación (Junca et al., 2002). Esta importancia se refleja en las exportaciones, que aunque variables, son significativas, con un número de individuos en el 2007 equivalente al 5\% de las exportaciones colombianas de peces ornamentales, siendo los principales destinos Estados Unidos y Europa (Olaya et al., 2007; Mancera y Álvarez, 2008; Aya y Arias, 2011).

El trabajo que se presenta a continuación permite aportar conocimiento de la morfología y morfometría del cerebro de bagre tigrito (Pimelodus pictus), y de esta forma hacer una aproximación de la interacción sensorial de esta especie en el ambiente donde habita, lo que podría servir como base para posteriores estudios de conectividad e interacción entre los diferentes aferentes sensoriales y de control motor.

\section{MATERIALES Y MÉTODOS}

Se utilizaron diez individuos de la especie Pimelodus pictus con una longitud total promedio de 9,52 $\pm 0,88 \mathrm{~cm}$, longitud estándar promedio de $7,48 \pm 0,75 \mathrm{~cm}$ y con un peso promedio de 6,25 $\pm 1,97$ gr. Los individuos se obtuvieron de una bodega acuarista.

\section{Procesamiento de muestras}

Este trabajo se realizó en el Laboratorio de Embriología del Campus Nueva Granada, Cajicá-Colombia. El procedimiento se llevó a cabo según las guías para el manejo ético de peces para la investigación (ASIH, 1988; AVMA, 2001; CCAC, 2005). Los individuos fueron anestesiados con benzocaína a una concentración de 0,1 g/l, hasta que no presentaran movimiento opercular (Gómez et al., 2011) y sacrificados por corte medular. Posteriormente, se retiró la cabeza del cuerpo y se realizó una abertura craneal; las cabezas se introdujeron en formaldehido al $4 \%$ ajustado a un $\mathrm{pH}$ de 7.2-7.3, en el cual se mantuvieron como mínimo cinco días a $4^{\circ} \mathrm{C}$ (Gómez et al., 2011). Pasado este tiempo se llevó a cabo la disección de las 
cabezas con el fin de obtener el cerebro, y así realizar la descripción morfológica y morfométrica.

\section{Descripción morfológica}

La identificación de las estructuras externas del cerebro se realizó con ayuda de imágenes de la parte dorsal, ventral y lateral de los cerebros. La captura de las imágenes se realizó con un estereoscopio (Carl Zeiss, Stemi DV4) equipado con una cámara digital. Posteriormente, se identificaron y compararon las estructuras presentes con la ayuda de los atlas de cerebro de algunos peces como: pez cebra, Danio rerio (Wullimann et al., 1996); medaka Oryzias latipes (Anken y Bourrat 1998; Ishikawa et al., 1999), anguila japonesa Anguilla japonica (Mukuda y Ando 2003), Dolloidraco longedorsalis (Eastmon y Lannoo, 1998), neón cardenal Paracheirodon axelrodi (Obando et al., 2013), lubina Dicentrarchus labrax
(Cerdá-Reverter et al., 2001), pez gato de canal Ictalurus punctatus (Grizzle y Rogers, 1976).

\section{Análisis morfométrico}

Una vez se obtuvieron las imágenes para la identificación de las estructuras externas, estas fueron procesadas y con el uso del Software ImageJ (<http:// rsbweb.nih.gov/ij/>) se tomaron medidas de área (Fig. 1) y longitud (Fig. 2) de ocho estructuras del cerebro: Bulbos olfativos (BO), hemisferios telencefálicos (HT), lóbulos ópticos (LO), corpus cerebelar (Ce), válvula cerebelar (Val), lóbulos vagales (LV), faciales (LF) e inferiores (LI) (Obando et al., 2013, Londoño et al., 2010). En el caso de las estructuras pareadas, se tomaron medidas de ambos lóbulos. Para realizar las medidas de área se tomaron imágenes tanto laterales como dorsales del cerebro (Obando et al.,2 013; Londoño et al., 2010).

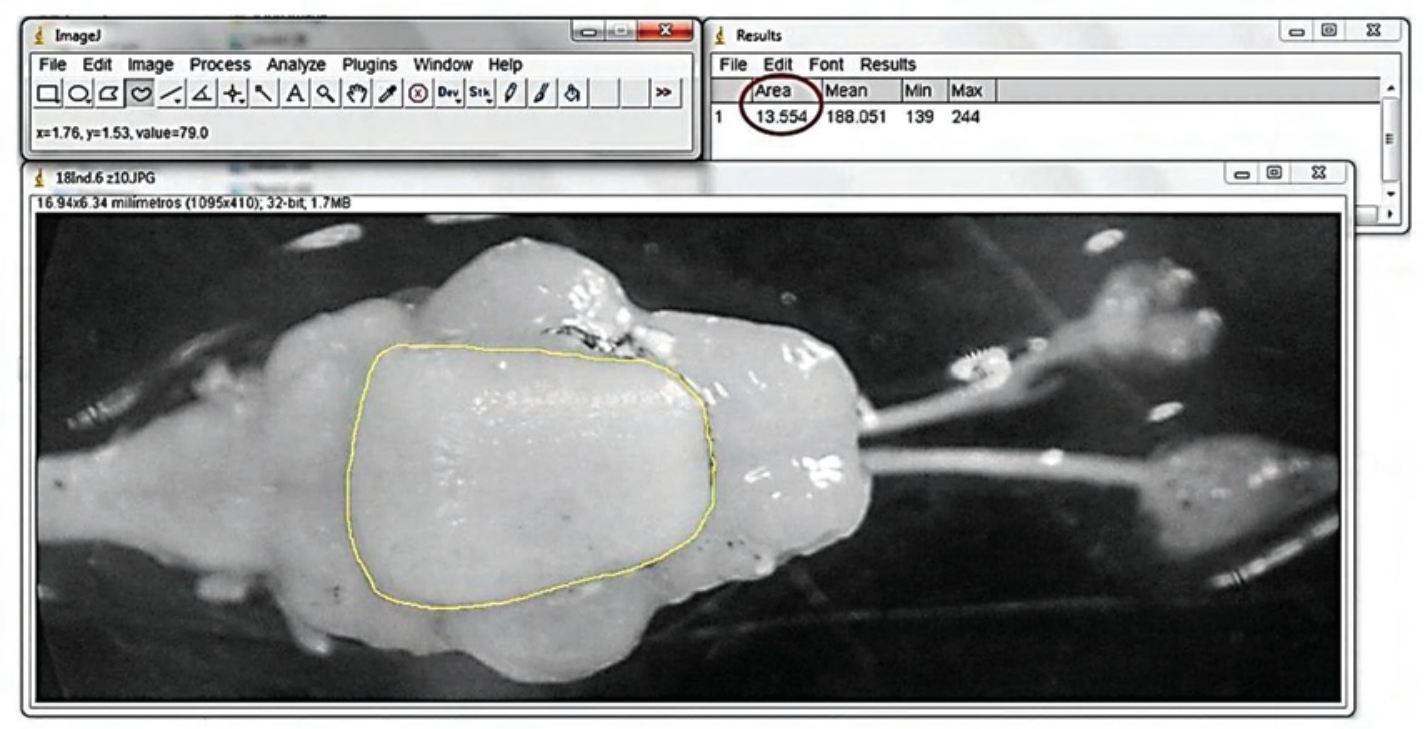

Figura 1. Ejemplo de medición del área de la Válvula cerebelar, a partir de imagen dorsal del cerebro de P. pictus 


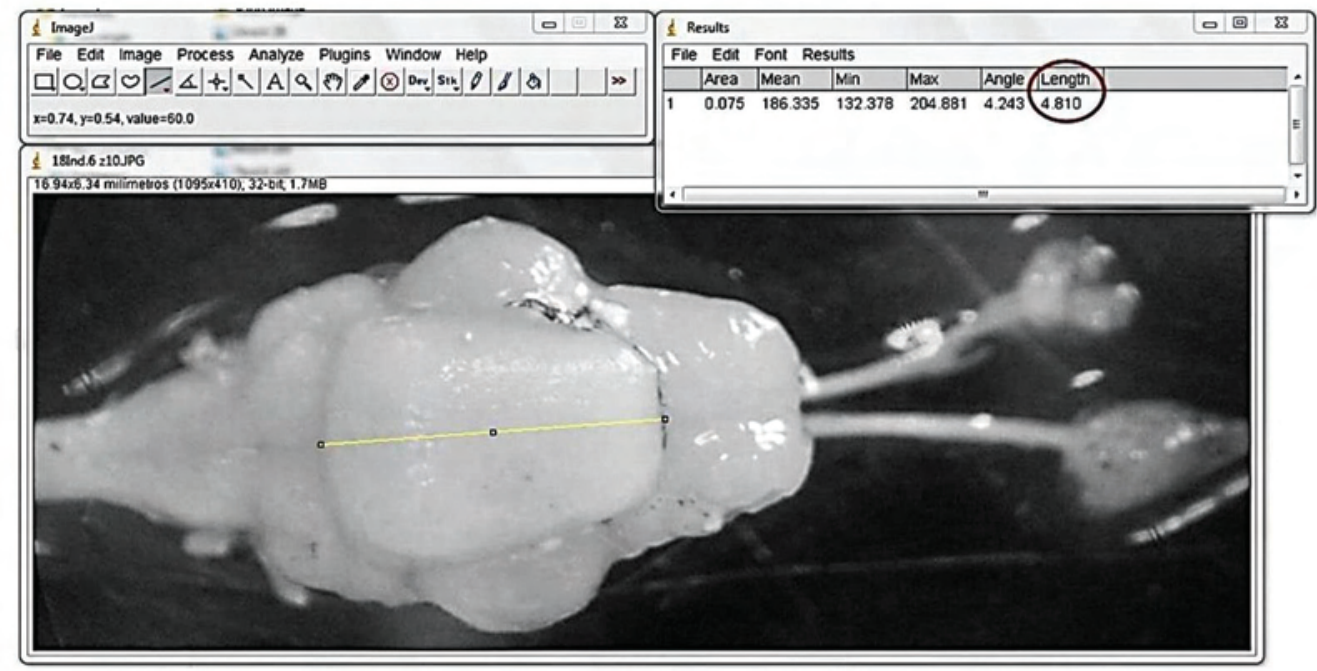

Figura 2. Ejemplo de medición de la longitud de la Válvula cerebelar, a partir de imagen dorsal del cerebro de P. pictus.

\section{Análisis estadístico}

Se realizó un análisis de varianza (ANOVA) y pruebas de Tukey para comprobar si existen diferencias significativas en área y longitud de las estructuras cerebrales evaluadas (bulbos olfativos, hemisferios telencefálicos, lóbulos ópticos, corpus cerebelar, válvula cerebelar, lóbulos vagales, faciales e inferiores). Se consideró el nivel de significancia del 5\% ( $p>0,05$ error tipo I) (Zar 2009). Para esto se empleó el paquete estadístico R versión 2.15.1 (http://www.r-project.org/).

\section{RESULTADOS}

\section{Descripción morfológica}

El cerebro de P. pictus ocupa la totalidad de la cavidad craneal presentando muy poco tejido adiposo a su alrededor (Fig. 3). Se identificaron varias estructuras en diferentes orientaciones del cerebro (dorsal, ventral y sagital) (Fig. 4 a 6).

En el cerebro de $P$. pictus se observaron los bulbos olfativos (BO), ubicados en la parte más rostral del cerebro, caudalmente se encuentran unidos a los hemisferios telencefálicos por medio de los tractos olfativos (TO). Los bulbos olfativos son pareados,

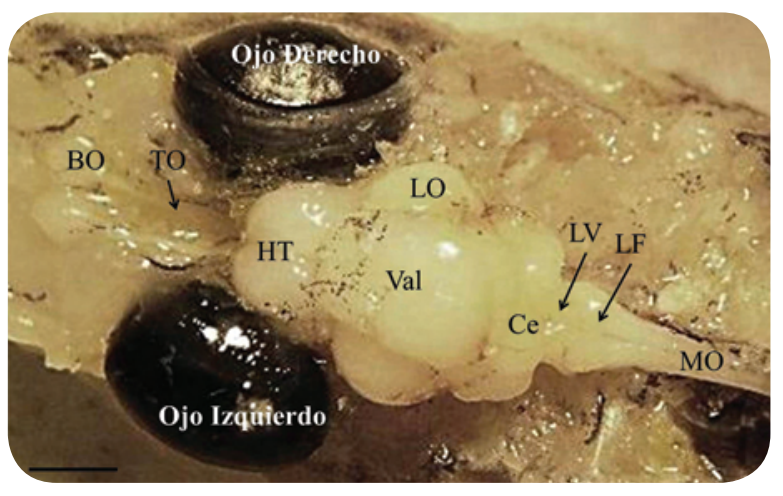

Figura 3. Vista dorsal del cerebro de P. pictus, ubicado sobre la zona ventral de la cavidad craneal. Bulbos olfativos (BO), Tractos olfativos (TO), Hemisferios telencefálicos (HT), Lóbulos ópticos (LO), Válvula cerebelar (Val), Corpus cerebelar (Ce), Lóbulos vagales (LV), Lóbulos faciales (LF), Médula oblonga (MO). Barra: $5 \mathrm{~mm}$.

con forma ovoide. Los hemisferios telencefálicos $(\mathrm{HT})$, localizados detrás de los tractos olfativos y delante de los lóbulos ópticos, con disposición pareada, presentan forma oblonga. Los lóbulos ópticos (LO) son pareados, se encuentran en una posición lateral, detrás de los hemisferios telencefálicos, presentan una forma circular y tanto dorsal como sagitalmente se evidencia una superposición del techo del cerebelo (Fig. 4, 5 y 6). 
El cerebelo o corpus cerebelar (Ce) (Fig. 4 y 6) presenta una forma irregular, formado por dos lóbulos circulares y se ubica caudal a los lóbulos ópticos. Sobre el corpus cerebelar se observa una proyección llamada válvula cerebelar (Val) que sobresale en la zona caudal a los lóbulos ópticos y se superpone dorsalmente sobre una parte de los mismos (Fig. 4, 5 y 6). Detrás del corpus cerebelar se encuentran los lóbulos vagales (LV) de forma ovoide.

Los lóbulos faciales (LF) (Fig. 4 y 6), son pareados y dispuestos en forma de "V". Cada lóbulo presenta una forma cilíndrica y se ubican en la región caudal del cerebro. Detrás de ellos se encuentra la medula oblonga (MO). Los lóbulos inferiores (LI) (Fig. 5 y 6) son pareados, se observan ventralmente, bajo los lóbulos ópticos. La hipófisis (Hip) (Fig. 5), está localizada en el vértice entre los lóbulos inferiores en posición rostral a éstos. Finalmente, la médula oblonga (MO) (Fig. 4, 5 y 6), localizada en la región más caudal del cerebro, con forma alargada, la cual continúa con la médula espinal.

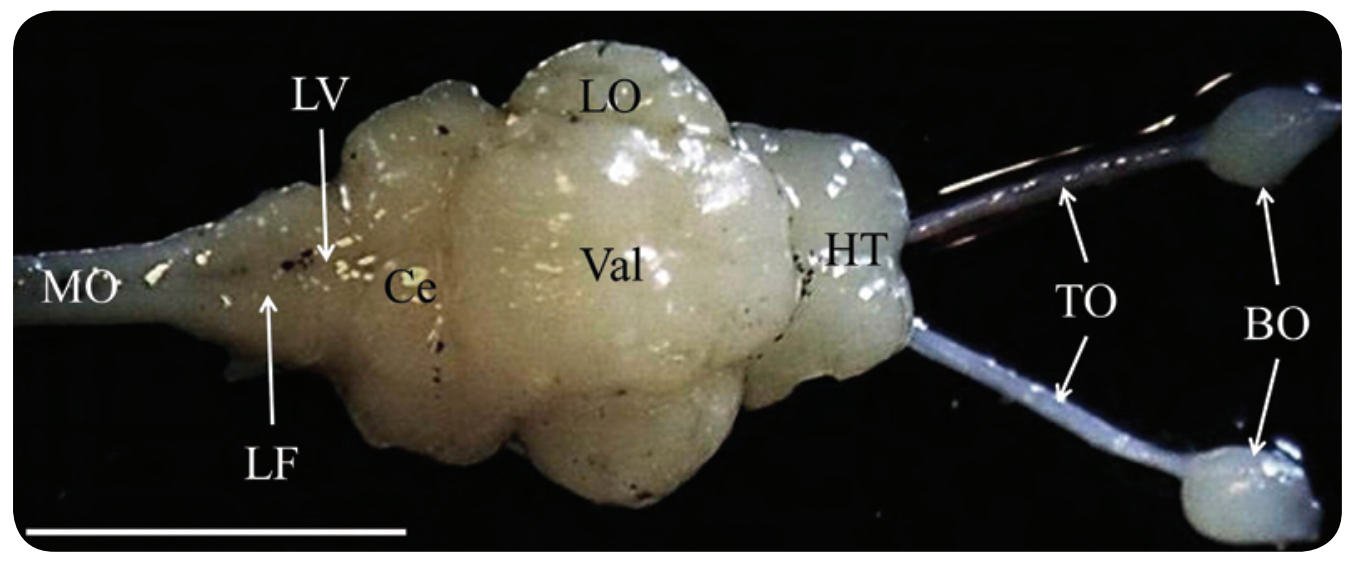

Figura 4. Vista dorsal del cerebro de P. pictus. Bulbos olfativos (BO), Tractos olfativos (TO), Hemisferios telencefálicos $(\mathrm{HT})$, Válvula cerebelar (Val), Corpus cerebelar (Ce), Lóbulos ópticos (LO), Lóbulos vagales (LV), Lóbulos faciales (LF), Médula oblonga (MO). Barra: $5 \mathrm{~mm}$.

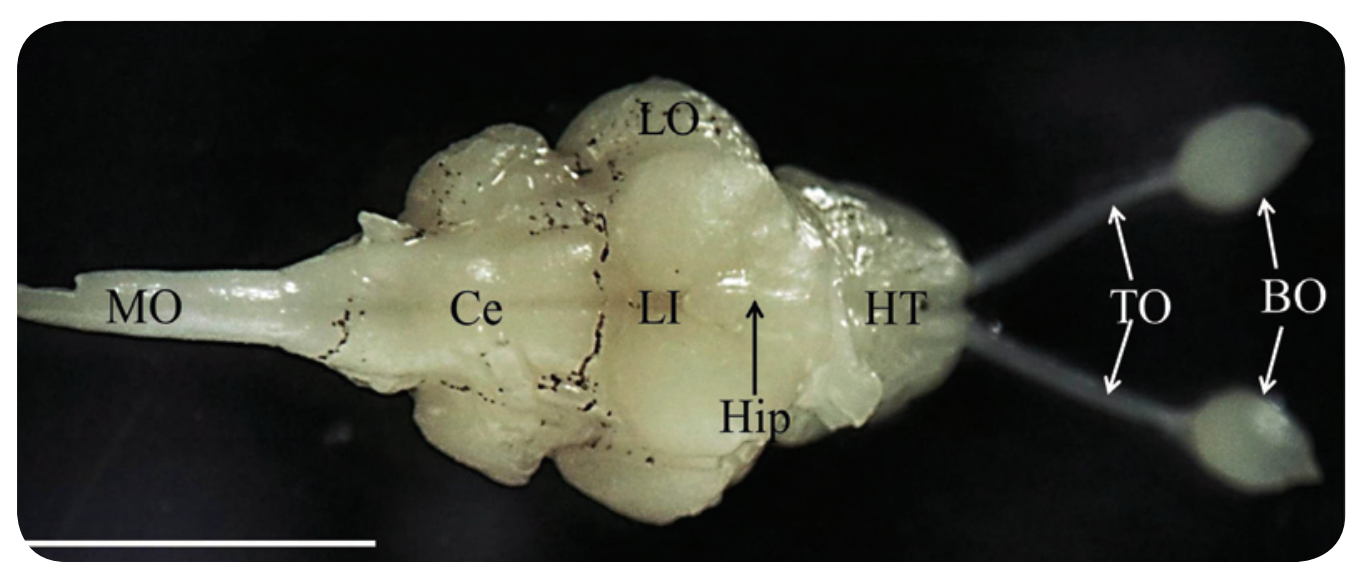

Figura 5. Vista ventral del cerebro de P. pictus. Bulbos olfativos (BO), Tractos olfativos (TO), Hemisferios telencefálicos (HT), Hipófisis (Hip), Lóbulos inferiores (LI), Lóbulos ópticos (LO), Corpus cerebelar (Ce), Médula oblonga (MO). Barra: 5 mm. 


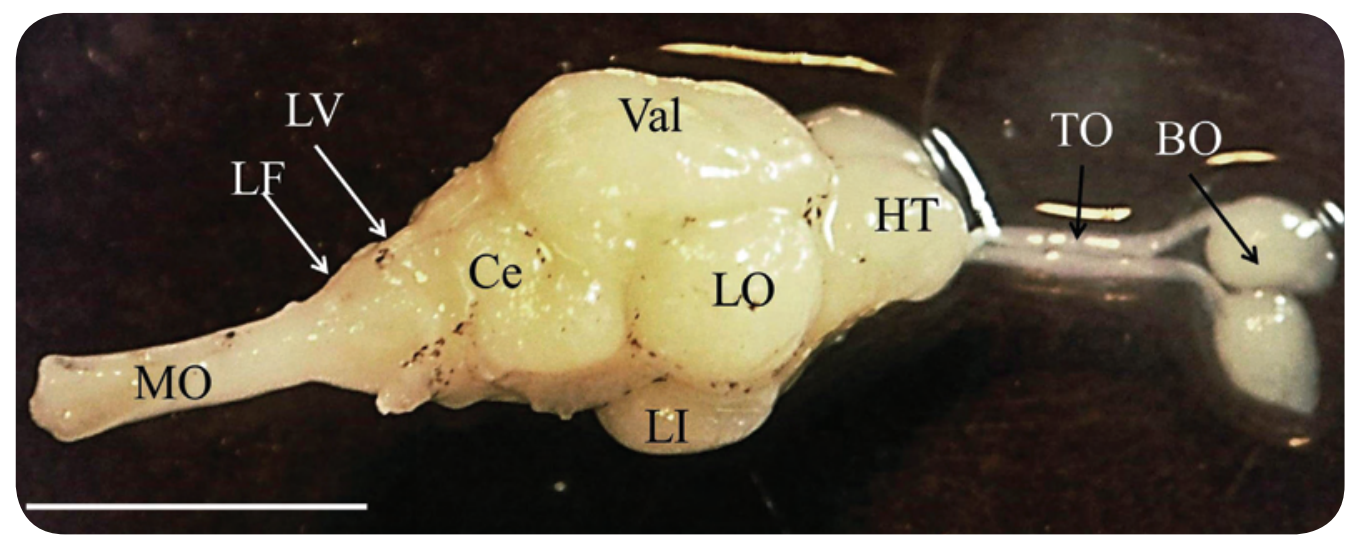

Figura 6. Vista lateral del cerebro de P. pictus. Bulbos olfativos (BO), Tractos olfativos (TO), Hemisferios telencefálicos (HT), Corpus cerebelar (Ce), Lóbulos ópticos (LO), Lóbulos inferiores (LI), Válvula cerebelar (Val), Lóbulos vagales (LV), Lóbulos faciales (LF), Médula oblonga (MO), Barra: $5 \mathrm{~mm}$.

\section{Análisis morfométrico}

La válvula cerebelar (Val) fue la región del cerebro que obtuvo el mayor valor de área $(13,75 \pm 0,30$ $\mathrm{mm}^{2}$ ), seguido de los lóbulos ópticos (LO) $\left(4,89 \pm 0,07 \mathrm{~mm}^{2}\right)$ y los hemisferios telencefálicos (HT) $\left(4,32 \pm 0,37 \mathrm{~mm}^{2}\right)$. Luego, con un área menor, encontramos los lóbulos inferiores (LI) $(2,66 \pm 0,1$ $\mathrm{mm})$, los bulbos olfatorios $(\mathrm{BO})\left(1,77 \pm 0,09 \mathrm{~mm}^{2}\right)$ y el corpus cerebelar (Ce) $\left(2,10 \pm 0,07 \mathrm{~mm}^{2}\right)$. Finalmente, las estructuras de menor área fueron los lóbulos faciales (LF) $\left(0,60 \pm 0,07 \mathrm{~mm}^{2}\right)$ y los lóbulos vagales (LV) $\left(1,02 \pm 0,07 \mathrm{~mm}^{2}\right)$. Para la variable de área se encontró que todas las estructuras cerebrales evaluadas son estadísticamente diferentes (Fig. 7).

La estructura de mayor longitud fue la válvula cerebelar (Val) $(4,69 \pm 0,13 \mathrm{~mm})$, seguido de los hemisferios telencefálicos $(H T)(2,79 \pm 0,07 \mathrm{~mm})$ y lóbulos ópticos (LO) $(2,89 \pm 0,06 \mathrm{~mm})$. Después seguían los lóbulos inferiores (LI) $(1,98 \pm 0,06 \mathrm{~mm})$, los cuales a su vez eran diferentes de los bulbos olfatorios (BO) $(1,54 \pm 0,04 \mathrm{~mm})$, el corpus cerebelar (Ce) $(1,60 \pm 0,08$ $\mathrm{mm}$ ) y los lóbulos faciales (LF) $(1,53 \pm 0,05 \mathrm{~mm})$. Por último la estructura de menor longitud fueron los lóbulos vagales (LV) $(1,02 \pm 0,07 \mathrm{~mm})$. Los hemisferios telencefálicos (HT) y lóbulos ópticos (LO), no eran estadísticamente diferentes entre sí, al igual que el

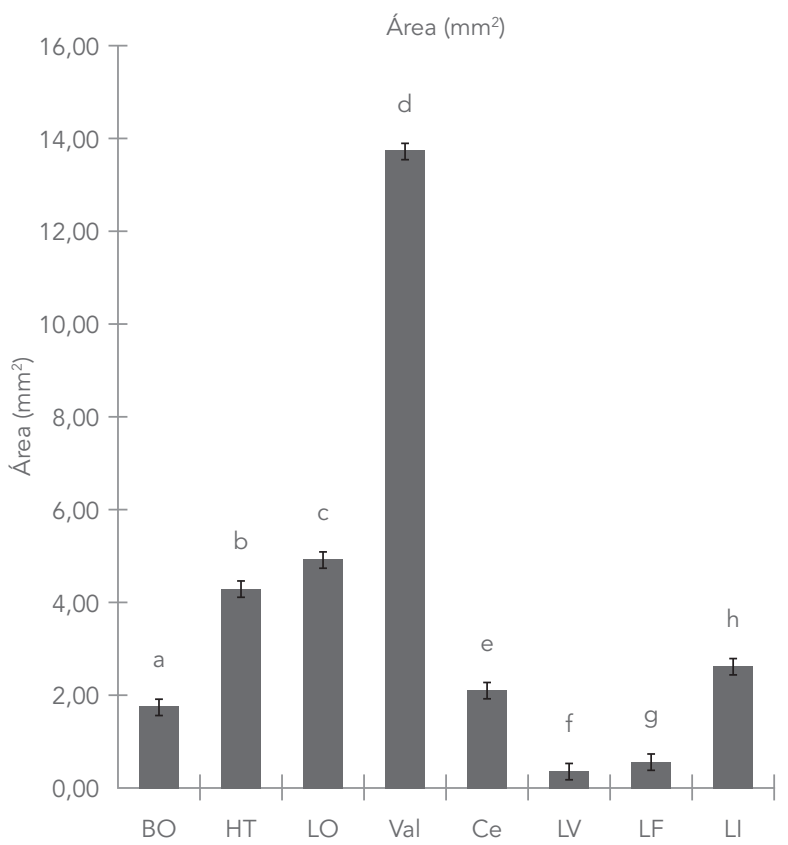

Figura 7. Área en milímetros cuadrados de las zonas del cerebro de P. pictus. Valores promedio. Cada valor representa el valor promedio \pm la desviación estándar del área de BO: bulbos olfativos; HT: hemisferios telencefálicos; LO: lóbulos ópticos; Ce: corpus cerebelar; Val: Válvula cerebelar; LV: lóbulos vagales; LF: lóbulos faciales; LI: lóbulos inferiores ( $n=20\{B O ; H T ; L O ; V a l ; L V ; L F ; L I\}, n=10$ $\{C \mathrm{e}\}, \alpha=0.05)$.

corpus cerebelar (Ce) y los lóbulos faciales (LF). El resto de estructuras cerebrales evaluadas son estadísticamente diferentes (Fig. 8). 


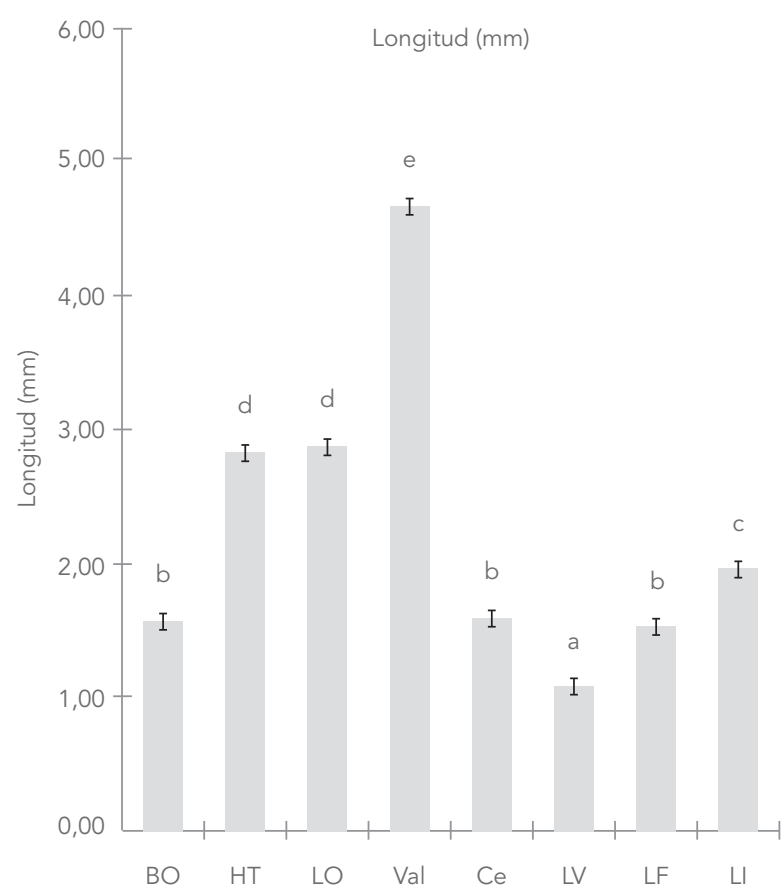

Figura 8. Longitud en milímetros de las zonas del cerebro de P. pictus. Cada valor representa el valor promedio \pm la desviación estándar de la longitud de BO: bulbos olfativos; HT: hemisferios telencefálicos; LO: lóbulos ópticos; Ce: corpus cerebelar; Val: Válvula cerebelar; LV: lóbulos vagales; LF: lóbulos faciales; LI: lóbulos inferiores ( $n=20\{B O ; H T$; LO; Val; LV; LF; LI\}, $n=10\{C e\}, \alpha=0.05)$.

\section{DISCUSIÓN}

Según el análisis morfológico realizado, el cerebro de $P$. pictus presenta un patrón de organización general típico de teleósteos (Cadwallader, 1975; Takashima y Hibiya, 1995; Wullimann et al., 1996; Wullimann, 1997; Kotrschal et al., 1998; Rose, 2002; Bălescu, 2005; Yoshida y Hirano, 2010). Dentro de las características destacadas en esta especie se evidenció una prolongación del cerebelo y la presencia de tractos olfativos, características compartidas con otras especies del orden Siluriformes como tiburoncito (Ariopsis seemanni) (Londoño et al., 2010) y capitán de la sabana (Eremophilus muttisi) (Quintero et al., 2009).

Éstas características morfológicas del cerebro de los Siluriformes difieren con otras especies de telósteos. Por ejemplo, en el orden Characiformes, el neón cardenal (Paracheirodon axelrodi) (Obando et al., 2013) y la cachama blanca (Piaractus brachypomus) (Forero y Hurtado, 2008) presentan bulbos olfativos sésiles, situados rostrales a los hemisferios telencefálicos sin presencia de tracto olfativo. Esta diferencia podría indicar una mayor velocidad de llegada de los estímulos olfatorios a los lóbulos olfatorios de los Characiformes, en comparación con los Siluriformes (Londoño et al., 2010).

El análisis morfométrico evidenció diferencias significativas en las medidas de área y longitud entre los distintos lóbulos que conforman el cerebro de $P$. pictus. Algunos autores reportan que el tamaño de las regiones del cerebro son un indicador de su grado de desarrollo, por esto es posible correlacionar los datos obtenidos con la influencia de éstas zonas cerebrales sobre las facultades sensoriales y su importancia en el comportamiento del individuo dentro del hábitat (Rose, 2002; Bălescu, 2005). Además, también se ha reportado que en los teleósteos hay una relación entre morfología y tamaño de las estructuras del cerebro, con parámetros comportamentales y ecológicos. Demostrando, que los cambios en las exigencias ambientales influyen fuertemente en el tamaño de los componentes en el cerebro pero no en su conectividad (Rose, 2002; Bălescu, 2005).

Morfométricamente, la zona del cerebro que presentó mayor área y longitud fue la válvula cerebelar (Val) seguido de los lóbulos ópticos (LO) y los hemisferios telencefálicos (HT). La válula cerebelar de $P$. pictus tiene características similares a la de los Osteoglossiformes, especialmente los mormíridos (peces eléctricos) y algunos Siluriformes, como el caso de A. seemanni (Área 2,3 $\pm 0,03 \mathrm{~mm}$; Longitud 6,1 $\pm 0,05 \mathrm{~mm}$ ) (Londoño et al.,2010), la cual se caracteriza por ser hipertrofiada y extenderse más allá del ventrículo tectal, por lo que cubre las superficies dorsales del corpus cerebelar y el tectum (Butler y Hodos, 1996; Wullimann y Rooney, 1990). 
La válvula cerebelar es un área altamente especializada en electrorrecepción y está relacionada con el funcionamiento de la línea lateral. Esto, gracias a que la válvula cerebelar se encuentra asociada a una región especializada del área octavolateralis, conocida como el lóbulo electrosensitivo de la línea lateral, el cual recibe la entrada electrosensorial (Butler y Hodos, 1996; Wullimann y Rooney, 1990). La válvula cerebelar es un órgano muy diferente en los peces no eléctricos, como goldfish (Cariasus auratus) y $P$. axelrodi, en el cual el corpus cerebelar es visible en el cerebro intacto y la válvula está escondida dentro del espacio del ventrículo tectal, debajo del tectum óptico (Butler y Hodos, 1996; Obando et al., 2013).

Los lóbulos ópticos (LO) fueron la tercera región con mayor tamaño en el cerebro de P. pictus. Esto coincide con el hecho de que es un pez con ojos relativamente grandes en proporción corporal, que habita en aguas lóticas principalmente oscuras (Huber et al.,1997; Pérez-Chaparro et al.,2001 y Junca et al.,2002). Se han realizado estudios que relacionan el tamaño del ojo con el hábitat de las especies y se ha determinado que peces con hábitos crepusculares y nocturnos presentan ojos de mayor tamaño (Pankhurst y Eagar, 1987; Eastmon y Lannoo 1998; Tovar et al.,2009b), como es el caso también de la trucha arcoíris (Oncorhynchus mykiss) (Tovar et al.,2008), E. mutisii (Tovar et al.,2008) y P. axelrodi (Tovar et al.,2009a; Obando et al.,2013). Por lo que los lobulos ópticos (LO) funcionan como un importante integrador visual, que también recibe e integra señales táctiles y auditivas e interviene en la locomoción y en la percepción espacial, por ello se le considera una región de integración multimodal (Takashima y Hibiya, 1995; Butler y Hodos, 1996).

Los hemisferios telencefálicos (HT) también presentaron un tamaño importante comparados con las demás estructuras, equivalente a lo reportado para

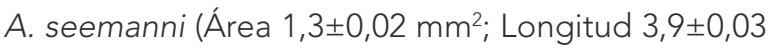
$\mathrm{mm}^{2}$ ) (Londoño et al.,2010). Según lo reportado, los hemisferios telencefálicos son una zona de procesamiento sensorio-motriz, teniendo conexiones visuales, auditivas y de la línea lateral. También, se relaciona con la recepción y transmisión de impulsos olfativos, alimentación, mecanismos asociados con procesos de memoria espacial, visión a color, aprendizaje, conducta reproductiva, agresiva y tareas sociales complejas (Kotrschal et al., 1998; Papini et al., 1999; Salas et al., 2006).

Huber et al., (1997) reportó que el tamaño de los hemisferos telencefálicos está relacionado con la habilidad de subsistir en ambientes espacialmente estructurados. Esto puede estar asociado con el hecho de que $P$. pictus habita preferiblemente lugares que presentan plantas, rocas o madera para esconderse (Pérez-Chaparro et al., 2001). Entonces, esta especie depende del aprendizaje espacial, teniendo en cuenta los ambientes en los que se desenvuelve y su característico comportamiento territorial, similar a lo reportado para C. auratus (Pérez-Chaparro et al., 2001; Cruz et al., 2010). Éstas características son factores que influyen favorablemente sobre el desarrollo de los hemisferios telencefálicos (Huber et al., 1997; Kotrschal et al., 1998; Papini et al., 1999; Salas et al., 2006).

Luego, con un área y longitud menor, encontramos los lóbulos inferiores (LI), los bulbos olfatorios (BO) y el corpus cerebelar (Ce). Los lóbulos inferiores (LI) están encargados de recibir entradas visuales de los núcleos pretectales, al igual que entradas gustativas y olfativas, por lo que parece estar muy involucrado con la percepción sensorial (Butler, 2000; Chiason y Radke, 1991). Este hecho está relacionado con la presencia de barbillones en P. pictus, los cuales son una entrada sensorial de gran importancia utilizados en la captura del alimento y en el reconocimiento espacial, permitiendo un mejor desenvolvimiento en los ambientes que habita la especie. Adicionalmente, los lóbulos inferiores están relacionados con actividades del sistema nervioso autonómico y el 
sistema endocrino, coordinando la producción de hormonas del sistema endocrino por medio de la hipófisis (Van Staaden et al., 1995; Butler y Hodos, 1996; Kotrschal et al., 1998; Eastmon y Lannoo, 1998; Kiyohara et al., 2002).

Los bulbos olfativos (BO), por su parte, están encargados de la recepción de estímulos relacionados con el sentido del olfato, y a su vez en la proyección de entradas olfativas a otras regiones del cerebro como la región ventral del telencéfalo y algunos núcleos del diencéfalo (Wullimann et al., 1996). De acuerdo con lo reportado por Chiason y Radke (1991), los peces que viven en lagunas o lagos con poca corriente presentan un modesto sentido del olfato y son dependientes de la visión y del gusto para ubicar el alimento, por lo que generalmente existe una posible oposición entre el sentido del olfato y el visual (Bauchot et al., 1982). P. pictus presenta lóbulos ópticos de gran tamaño en contraste con bulbos olfatorios de menor tamaño, lo cual podría decir, que esta especie está utilizando más la visión que el sentido del olfato, similar a lo reportado para A. seemanni (Londoño et al., 2010), E. muttisi (Quintero et al., 2009) y P. axelrodi (Obando et al., 2013). Esto también puede estar asociado con que $P$. pictus es una especie tiene hábitos crepusculares y que vive en aguas oscuras principalmente (Pérez-Chaparro et al., 2001; Junca et al., 2002; Rodríguez y Mojica, 2005).

En cuanto al corpus cerebelar (Ce), Cadwallader (1975) reportó la correspondencia entre esta estructura y la presencia de los individuos en ambientes de aguas lóticas o lénticas, encontrando que los peces que habitan en aguas lóticas presentan cerebelos de mayor proporción, ya que entre mayor es la fuerza de la corriente, los individuos deben ejercer un mayor control motor, requiriendo movimientos rápidos con mayor precisión, actividades que son reguladas por el cerebelo. Esto se relaciona con el hecho de que $P$. pictus es una especie que habita en aguas lóticas, principalmente oscuras, que tiene hábitos crepusculares y que en su dieta incluye presas móviles por lo que requiere de movimientos rápidos y coordinados para desenvolverse en su hábitat (Pérez-Chaparro et al., 2001; Junca et al., 2002; Rodríguez y Mojica, 2005). Esto repercute en el hecho de que ésta especie tenga un cerebelo y una válvula cerebelar de gran tamaño.

Los lóbulos vagales (LV) y faciales (LF) presentan un área y longitud menor en comparación con el resto de estructuras cerebrales, pero en comparación con otras especies presentan un tamaño considerable. Dicho desarrollo también se observa en otras especies del orden Siluriformes, como por ejemplo A. seemanni, Lóbulos vagales (Área 0,2 0,01 mm²; Longitud 1,1 $\pm 0,02 \mathrm{~mm}^{2}$ ) y Lóbulos faciales (Área 0,3 $\pm 0,01 \mathrm{~mm}^{2}$; Longitud 1,1 $\pm 0,01 \mathrm{~mm}^{2}$ ) (Castañeda et al., 2013 y Londoño et al.,2010) y E. muttisi (Quintero et al., 2009) las cuales son especies que se caracterizan por la presencia de barbillones.

Los barbillones son órganos sensoriales, con terminaciones nerviosas altamente desarrolladas en botones gustativos, receptores de señales cutáneas y gustativas extraorales, que son innervados por ramas del nervio facial (Van Staaden et al., 1995; WuIlimann et al., 1996; Kotrschal et al., 1998; Eastmon y Lannoo 1998; Kiyohara et al., 2002). Es por ello que los lóbulos faciales y vagales en estos individuos presentan un mayor desarrollo, en comparación con especies que no cuentan con barbillones como es el caso de C. auratus (Cruz et al., 2010) y P. axelrodi (Obando et al., 2013).

Wagner (2001a), en su estudio sobre áreas del cerebro en peces Abisal-demersales, reportó que el $40 \%$ de las especies de peces utiliza primordialmente un sistema sensorial (especialistas), el 46\% utiliza dos sistemas sensoriales (dominantes) y el 14\% restante actúa bajo tres sistemas sensoriales (generalistas). De acuerdo a los resultados morfométricos obtenidos del cerebro de P. pictus, esta especie podría 
clasificarse como generalista, debido a que parece utilizar tres sistemas sensoriales principalmente: el tacto, la vista y el gusto. El tacto, ya que está especie presentó una valvula cereberal de gran tamaño, la cual está relacionada directamente con la presencia de la línea lateral, que es considerada un órgano sensorial cutáneo. La visión, teniendo en cuenta que los lóbulos ópticos de $P$. pictus presentaron un gran tamaño en comparación con otras estructuras cerebrales, y que los ojos de esta especie son de un tamaño considerable en relación con el tamaño corporal, lo que puede deberse a una especialización visual de esta especie de acuerdo con el hábitat en el que se desenvuelve. Y por último, el gusto, porque aunque los lóbulos vagales y faciales no presentaron un tamaño representativo en cuanto a su morfometría en comparación con las otras estructuras cerebrales, sí son grandes en comparación con otras especies de peces teleósteos, esto por la presencia de barbillones, las cuales son estructuras especializadas en integrar señales cutáneas y gustativas.

A manera de conclusión, se puede decir que la morfología del encéfalo de P. pictus presentó el patrón general de teleósteos del orden Siluriforme, siendo la válvula cerebelar la estructura más grande del cerebro y sugiriendo según la morfología y morfometría presentada que esta especie utiliza el tacto, la vista y el gusto principalmente para explotar su hábitat.

\section{AGRADECIMIENTOS}

Agradecemos el apoyo financiero por parte de la Universidad Militar Nueva Granada al proyecto CIAS-936.

\section{BIBLIOGRAFÍA}

1. ASIH (American Society of Ichthyologists and Herpetologists, American Fisheries Society, American Institute of Fisheries Research Biologists). 1988. Guidelines for use of fishes in field research. Fisheries, 13 (2): 16-23.

2. Anken R y F Bourrat. 1998. Brain Atlas of the Medaka fish Oryzias latipes. Institut National de la Recherche Agronomique. Paris. 29p.

3. AVMA (American Veterinary Medical Association). 2000. Report of the AVMA panel on euthanasia. JAVMA, 218 (5): 669-696.

4. Aya B y C Arias. 2011. Reproducción inducida de Pimelodus pictus con extracto de hipófisis de carpa (EHC) y Ovaprim ${ }^{\circledR}$. Revista Mvz Córdoba, 16 (1): 2317-2323.

5. Bălescu C. 2005. A Comparative Study on the External Morphology of the Encephalon in Three Fish Species Belonging to Cyprinidae Family: Amblypharyngodon mola, Danio malabaricus and Rasbora argyrotaenia. Analele Ştiintifice ale Universităţii. p 45-50.

6. Bauchot R, M Diagne y J Ridet. 1982. The brain of Photoblepharon palpebratus steinitzi (Pisces, Teleostei, Anomalopidae). Journal of Hirnforsch. 23 (4): 399-404.

7. Butler A. 2000. Nervous system, p: 131-141. En: Ostrander GK, Eds. The Handbook of Experimental Animals: The Laboratory Fish. Academic Press, California, 678p.

8. Butler A y W Hodos. 1996. Comparative Vertebrate Neuroanatomy: Evolution and Adaptation. John Wiley \& Sons Inc, New York, 552p 
17. Forero $\mathrm{C}$ y H Hurtado. 2008. Análisis histomorfológico preliminar del cerebro en alevinos de Cachama blanca, Piaractus brachypomus (Characiformes: Characidae). Trabajo de grado. Programa de Biología Aplicada. Facultad de Ciencias y Aplicadas. Universidad Militar Nueva Granada. Cajicá-Cundinamarca. 87p.

18. Galvis G, JI Mojica y M Camargo. 1997. Peces del Catatumbo. Ecopetrol-Oxy-Shell-Asociación Cravo Norte. D’Vinni Edit Ltda. Bogotá, 188p.

19. Gómez E, MJ Obando, MO Tovar, ML Caldas y H Hurtado. 2011. Estudio histológico del tracto digestivo del Neón Cardenal Paracheirodon axelrodi (Characidae). International Journal of Morphology, 29 (3):782-786.

20. Grizzle J y W Rogers. 1976. Anatomy and Histology of the Channel Catfish. Auburn Printing, Inc. Alabama, 94p.

21. Huber R, MJ Van Staaden, LS Kaufman y KF Liem. 1997. Microhabitat use, trophic patterns, and the evolution of brain structure in African cichlids. Brain, Behavior and Evolution, 50 (3): 167-182.

22. Ishikawa $\mathrm{Y}, \mathrm{M}$ Yoshimoto, $\mathrm{N}$ Yamamoto y $\mathrm{H}$ Ito. 1999. Different brain morphologies from different genotypes in a single teleost species, the medaka (Oryzias latipes). Brain, Behavior and Evoluttion, 53 (1): 2-9.

23. Iwaniuk AN. 2010. Comparative Brain Collections are an Indispensable Resource for Evolutionary Neurobiology. Brain, Behavior and Evoluttion, 76 (2): 87-88.

24. Junca R, A Vallejo, A Molano, A Pinilla. 2002. Fecundidad en el Tigrito Pimelodus pictus 
(Steindachner, 1876) (Pisces: Siluriformes: Pimelodidae). Boletín científico INPA, 7: 33-48.

25. Kiyohara S, Y Sakata, T Yoshitomi, J Tsukuhara. 2002. The "goatee" of Goatfish: Innervation of Taste Buds in the Barbels and their Representation in the Brain. Proceedings. Biological Sciences, 269(1502):1773-1780.

26. Kotrschal K, MJ Van Staaden y R Huber. 1998. Fish brains: evolution and environmental relationships. Reviews in Fish Biology and Fishiers, 8: 373-408.

27. Londoño C y H Hurtado. 2010. Estudio preliminar morfológico y morfométrico de encéfalo del pez tiburoncito, Ariopsis seemanni (Pisces: Ariidae). Universitas Scientiarum, 15 (2): 101-109.

28. Mancera N y R Álvarez. 2008. Comercio de Peces Ornamentales en Colombia. Acta Biológica Colombiana, 13: 23-52.

29. Mondragón AM, D Becerra, AC Torres, ML Cifuentes, H Hurtado, E Gomez. En prensa. Evaluación de Sistema Cerrado de Recirculación para el Levante de Bagre tigrito (Pimelodus pictus).

30. Moreno VL, CJ Arias y BE Aya. 2008. Desarrollo ovocitario de Pimelodus pictus. Revista Colombiana de Ciencias Pecuarias, 21:455-522.

31. Mukuda Ty M Ando. 2003. Brain atlas of the Japanese Eel: comparison to other fishes. Memoirs of the Faculty of Integrated Arts and Sciences, 29: 1-25.

32. Obando MJ, E Gómez, MO Tovar, L Rincón, ML Caldas y H Hurtado. 2013. Estudio morfométrico y topológico del cerebro del pez Neón Cardenal, Paracheirodon axelrodi (Characiformes: Characidae). Actualidades Biológicas, 35 (98): 45-61.
33. Olaya CM, CH Ovalle, E Gómez, D Rodríguez, ML Caldas y H Hurtado. 2007. Histología y Morfometría del Sistema Digestivo del Silúrido Bagre tigrito (Pimelodus pictus). Revista de la Facultad de Medicina Veterinaria y de Zootecnia. 54: 311-323.

34. Pankhurst PM y R Eagar. 1996. Changes in visual morphology through life history stages of the new Zealand snapper, Pagrus auratus. New Zealand Journal of Marine \& Freshwater Research, 30: 70-90.

35. Papini MR, C Salas y RN Muzio. 1999. Análisis Comparativo del Aprendizaje en Vertebrados. Revista Latinoamericana de Psicología, 31 (1): 15-34.

36. Pérez-Chaparro LB, RE Ajiaco-Martínez y H Ramírez-Gil. 2001. Pimelodus pictus Steindachner 1876, p: 203-205. En: Ramírez-Gil H y RE Ajiaco Martínez (Eds). La pesca en la baja Orinoquía colombiana: Una visión integral. Minagricultura/ Pronatta/Colciencias/Inpa. Bogotá, 255p.

37. Quintero VM, RJ Bonilla, E Gómez, D Rodríguez, GH Hurtado. 2009. Distribución de Botones Gustativos en los Barbillones del Capitán de la Sabana (Eremophilus mutissi). Revista Facultad de Ciencias Básicas, 5: 186-191.

38. Rodríguez JA y HO Mojica. 2005. Reproducción y Manejo de Silúridos en Cautiverio, p: 105-122. En: Daza P, MA Landines y Al Sanabria. Reproducción de los Peces en el Trópico. Instituto Colombiano de Desarrollo Rural- INCODER. Universidad Nacional de Colombia, Facultad de Medicina Veterinaria y Zootecnia, Bogotá, 246p.

39. Rose JD. 2002. The Neurobehavioral Nature of Fishes and the Question of Awareness and Pain. Reviews in Fisheries Science, 10 (1): 1-38. 
40. Salas C, C Broglio, E Durán, A Gómez, FM Ocaña, F Jiménez-Moya y F Rodríguez. 2006. Neuropsychology of Learning and Memory in Teleost Fish. Zebrafish, 3 (2): 157-171.

41. Takashima F y T Hibiya. 1995. An atlas of fish histology: Normal and pathological features. 2ed. Kodansha, Tokyo. 147p.

42. MO Tovar, LF Contreras, ML Caldas, D Rodríguez Caicedo y H Hurtado. 2008. Comparación Histológica y Morfométrica entre el Ojo de Eremophilus mutisii (Trichomycteridae) y el de Oncorhynchus mykiss (Salmonidae). Acta Biológica Colombiana, 13 (2): 73-88.

43. Tovar MO, MJ Obando, E Gómez, ML Caldas y H Hurtado. 2009a. Histología y morfometría del ojo del pez dulceacuícola Paracheirodon axelrodi (Characiformes: Characidae). Revista de Biología Tropical, 57 (4): 1107-1118.

44. Tovar MO, SJ Corchuelo, DC Castañeda y H Hurtado. 2009b. El Concepto de Ecología Visual. Revista Facultad de Ciencias Básicas, Universidad Militar Nueva Granada, 5 (1): 24-41.

45. Van Staaden MJ, R Huber, LS Kaufman y KF Liem. 1995. Brain evolution in Cichlids of the African Great Lakes: brain and body size, general patterns, and evolutionary trends. Zoology 98:165-178.

46. Wagner HJ. 2001a. Brain Areas in Abyssal Demersal Fishes. Brain, Behavior and Evolution, 57 (6): $301-316$.
47. Wagner HJ. 2001b. Sensory Brain Areas in Mesopelagic Fishes. Brain, Behavior and Evolution, 57 (3): 117-133.

48. Wullimann MF y DJ Rooney. 1990. A Direct Cerebello-Telencephalic Projection in an Electro Sensory Mormyrid Fish. Brain Research, 520 (1-2): 354-357.

49. Wullimann MF, B Rupp y H Reichert. 1996. Neuroanatomy of the Zebrafish Brain: A Topological Atlas. Birkhaeuser Verlag, Switzerland, 190p.

50. Wullimann MF. 1997. Major Patterns of Visual Brain Organization in Teleosts and their Relation to Prehistoric Events and the Paleontological Record. Paleobiology, 23 (1): 101-114.

51. Yoshida M y R Hirano. 2010. Effects of Local Anesthesia of the Cerebellum on Classical Fear Conditioning in Goldfish. Behavioral and Brain Functions, 6 (20): 1-9.

52. Zar HJ. 2009. Biostatistical Analysis. 5ed. Editorial Pearson Education, New Jersey, 944 p.

\section{CONSULTA PAGINAS WEB}

1. ImageJ. 2011. National Institutes of Health. Disponible en: http://rsbweb.nih.gov/ij/download. html. Consultada el 19 de agosto de 2011.

2. R-Project 2.15.1. Disponible en: http://www.rproject.org/. Consultada el 20 de marzo de 2011. 